\title{
Boundary control of a nonlinear Stefan Problem
}

\author{
William B. Dunbar \\ Control and Dynamical Systems \\ California Institute of Technology \\ Mail Code 107-81, 1200 E California Blvd \\ Pasadena, CA 91125, USA \\ dunbarecds.caltech.edu
}

\author{
Nicolas Petit*, Pierre Rouchon, Philippe Martin \\ Centre Automatique et Systèmes \\ École Nationale Supérieure des Mines de Paris \\ 60, bd. Saint-Michel \\ 75272 Paris Cedex 06, France \\ petit, rouchon, martin \}eensmp.fr
}

\begin{abstract}
The classical Stefan problem is a linear onedimensional heat equation with a free boundary at one end, modelling a column of liquid (e.g. water) in contact with an infinite strip of solid (ice). Given the fixed boundary conditions, the column temperature and free boundary motion can be uniquely determined. In the inverse problem, one specifies the free boundary motion, say from one steady-state length to another, and seeks to determine the column temperature and fixed boundary conditions, or boundary control. This motion planning problem is a simplified version of a crystal growth problem. In this paper, we consider motion planning of the free boundary (Stefan) problem with a quadratic nonlinear reaction term. The treatment here is a first step towards treating higher order nonlinearities as observed in crystal growth furnaces. Convergence of a series solution is proven and a detailed parametric study on the series radius of convergence given. Moreover, we prove that the parametrization can indeed be used for motion planning purposes; computation of the open loop motion planning is straightforward and we give simulation results.
\end{abstract}

Keywords: Distributed parameter system, boundary control, inverse Stefan problem, flatness.

\section{INTRODUCTION}

In this paper we consider a free boundary problem for a nonlinear parabolic partial differential equation. In particular, we are concerned with the inverse problem, which means we know the behavior of the free boundary a priori and would like a solution, e.g. a convergent series, in order to determine what the trajectories of the system should be for steady-state to steady-state boundary control.

The classical Stefan problem models a column of liquid in contact at 0 degrees with an infinite strip of its solid phase, as depicted in Figure 1. The problem is thoroughly explored in [1] and a catalogue of various problems reducing to problems of the Stefan type is given in [15]. We investigate a modified Stefan problem that includes a diffusion term and a nonlinear reaction term. This can be seen as a simple model of a chemically reactive and heat diffusive liquid surrounded by ice, as considered under a more general form in [3].

Define $(x, t) \mapsto u(x, t)$ as the temperature in the liquid and $t \mapsto y(t)$ as the position of the liquid/solid interface, given a position $x$ and time $t$. The functions $h(t)$ and $\psi(x)$ are the temperatures at the fixed end $(x=0)$ and at initial time $(t=0)$, respectively. The nonlinear Stefan problem is to determine a $u(x, t)$ and $y(t)$, given $h(t)$ and $\psi(x)$, that satisfy

$$
\left.\begin{array}{ll}
u_{t}=u_{x x}-\nu u_{x}-\rho u^{2}, & \forall(x, t) \in D_{T} \\
u(0, t)=h(t) \geq 0, & 0<t \leq T \\
u(x, 0)=\psi(x) \geq 0, & 0 \leq x \leq y(0) \\
u(y(t), t)=0, u_{x}(y(t), t)=-\dot{y}(t), & 0<t \leq T
\end{array}\right\}
$$

where

$$
D_{T} \equiv\{(x, t): 0<x<y(t), 0<t \leq T\}
$$

and the boundaries are denoted in order as

$$
\begin{aligned}
B_{T} \equiv & \{(0, t): 0<t \leq T\} \cup\{(x, 0): 0 \leq x \leq y(0)\} \\
& \cup\{(y(t), t): 0<t \leq T\} \equiv B_{T}^{1} \cup B_{T}^{2} \cup B_{T}^{3} .
\end{aligned}
$$

As in [1], we refer to $D_{T}$ and $B_{T}$ as the parabolic interior and parabolic boundary, respectively. Figure 2 gives a graphical 2-D representation of the interior and boundary.

The notation $\dot{y}(t)$ is the time derivative of $y(t)$ and $\nu$, $\rho \geq 0, T>0$. This model arises from a classical energy balance. The equation $u_{x}(y(t), t)=-\dot{y}(t)$ expresses the fact that all of the heat energy arriving at the liquid-solid interface is utilized in the melting process. In the model, the thermal conductivity coefficient and a parameter equal to the product of the solid density and the latent heat of fusion are normalized to one. Of course it is possible to use different values for these coefficients using changes of scales for $x, t$ and $u$, as described in [1, page 282].

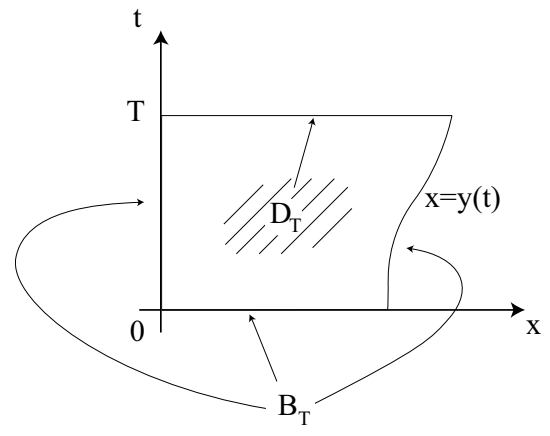

Fig. 2. Picture of parabolic interior and boundary for free boundary problem

The inverse problem and its solution are stated here as a definition. 


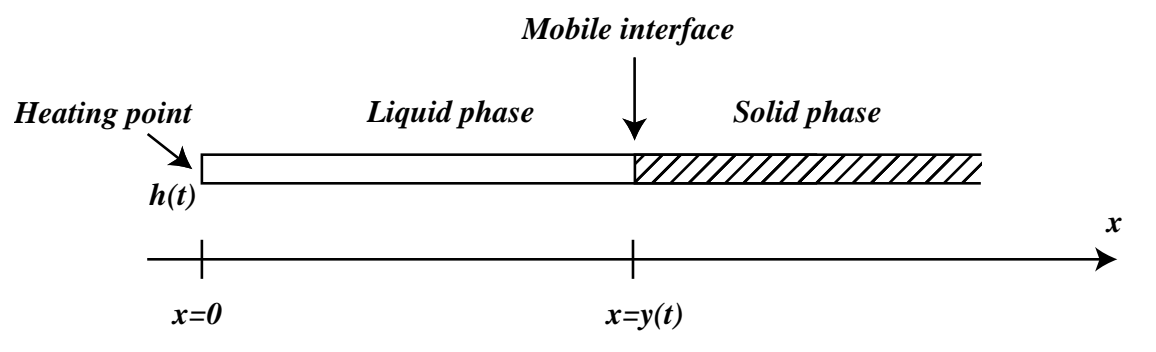

Fig. 1. The system under consideration: liquid phase governed by a nonlinear heat equation with boundary control, in contact with an infinite solid phase.

Definition 1: A solution of (1) for a known function $y \in$ $C^{\infty}[0, T]$, with all derivatives known, is a function $u=$ $u(x, t)$ defined in $D_{T} \cup B_{T}$ such that $u_{x x}, u_{t} \in C\left(D_{T}\right), u$ is bounded, satisfies the conditions of (1) and $u \in C\left(D_{T} \cup B_{T}\right)$. The inverse problem is precisely a non-characteristic Cauchy problem with Cauchy data: $u(y(t), t)=0, \quad u_{x}(y(t), t)=$ $-\dot{y}(t)$ [8]. Given $y(t)$ and a solution $u$ to the inverse problem, we have the initial profile $\psi(x)$ and the boundary control $h(t)$, both of which must be non-negative according to (1).

The flatness approach [5], [4] for partial differential equations are a means of solving inverse problems. The explicit parametrization of the trajectories of the systems is the key to straightforward motion planning strategies that can incorporate optimization [12], [13].

Recent work on these parametrization include [11], [10] where approximate controllability of any initial condition to steady state is explored. The series expansion techniques used for heat equations have been used since the work of Gevrey, while inverse problems have been addressed as early as the work by Hill [8]. Specifically, Hill gives a complete solution to the inverse Stefan problem with a linear heat equation, i.e. $u_{t}=u_{x x}$. Recently a nonlinear heat equation over a constant spatial domain, with a quadratic reaction term, was examined by Rudolph and Lynch [11].

In this paper we combine the two issues: the free boundary (Stefan) problem with a quadratic nonlinearity. Using the work in [11] as a starting point, we prove convergence of a series solution. Then a parametric study on the series radius of convergence is carried out. Moreover, we prove that the parametrization can indeed can be used for motion planning purposes; computation of the open loop motion planning is straightforward. We also give brief simulation results.

The Stefan problem we consider is a first step towards a more complex problem for multidimensional reactiondiffusion systems investigated in [6], where three chemical species balance equations contain second order reaction terms. Extension of our approach to a fourth order radiation term, i.e. the Stefan-Boltzmann condition, is a subject for future work. A model with this condition arises in crystal growth furnaces, where a solid phase is surrounded by an infinite liquid phase. We feel the work presented here can highlight some of the difficulties and challenges of problems that arise in motion planning for crystal growth models.

\section{Series Solutions And Convergence}

\section{A. Outline}

In Section II-B we establish a lower bound on the radius of convergence of a series solution, denoted $\eta^{*}$, that depends upon the physical constants of the model $\rho$ and $\nu$. The radius $\eta^{*}$ also depends upon $M$ and $R$, the Gevrey constants of the function $\dot{y}(t)$. The definition of a Gevrey function is given implicitly in Theorem 1 here (and also in [9]), from which it is clear that the associated constants ( $M$ and $R$ in this case) characterize the aggressiveness of the trajectories of the system. In Section II-C we make use of several lemmas to construct a parametric lower bounds on the radius. The bound can be used in practice to guarantee convergence over a given domain.

\section{B. Series Solution}

Consider the series solution

$$
u(x, t)=\sum_{n=0}^{\infty} \frac{a_{n}(t)}{n !}[x-y(t)]^{n} .
$$

For the solution (2) to satisfy

$$
u_{t}=u_{x x}-\nu u_{x}-\rho u^{2}, \quad \forall(x, t) \in D_{T},
$$

the $a_{n}(t)$ coefficients must satisfy the recurrence equation

$$
a_{n}=\dot{a}_{n-2}-a_{n-1} \dot{y}+\nu a_{n-1}+\rho \sum_{k=0}^{n-2}\left(\begin{array}{c}
n-2 \\
k
\end{array}\right) a_{n-2-k} a_{k}
$$

where $n \geq 2$, with $a_{0}=0$ (arising from the $u(y(t), t)=0$ condition) and $a_{1}=-\dot{y}$ (arising from the Stefan condition $\left.-u_{x}(y(t), t)=\dot{y}(t)\right)$. These necessary conditions are also sufficient. From (3) it is clear that given $y(t)$, all the series coefficients $a_{n}(t)$ and therefore the temperature $u(x, t)$ and boundary conditions $h(t)$ and $\psi(x)$ are uniquely determined.

By majorizing the series in (2), we will prove that this solution converges absolutely. We now state the first of two 
main theorems in the paper. The proof makes use of two lemmas stated in the appendix.

Theorem 1: Given that $\dot{y} \in C^{\infty}[0, T]$ is Gevrey of order $(\alpha-1)$ for $1 \leq \alpha \leq 2$, i.e.

$$
\begin{aligned}
& \exists M, R>0 \quad \text { such that } \\
& \left|y^{(l+1)}(t)\right| \leq M \frac{l !^{\alpha}}{R^{l}}, \forall l=0,1,2, \ldots, \forall t \in[0, T],
\end{aligned}
$$

the radius of convergence of the series has as a lower bound the unique positive root $\eta=\eta^{*}$ of the polynomial

$$
\left(\frac{\rho M}{2}\right) \eta^{3}+\left(\frac{1}{R}\right) \eta^{2}+\left(\frac{\nu+M}{2}\right) \eta-1=0 .
$$

Proof: By induction on $n$, we prove that for all $n=$ $0,1,2, \ldots$, the coefficients satisfy the bound

$$
\left|a_{n}^{(l)}(t)\right| \leq \frac{M A^{n-1}}{R^{l+n-1}} \frac{(l+n) !^{\alpha}}{n !^{\alpha-1}}, \forall l=0,1,2, \ldots
$$

for some $A>0$. The coefficient $a_{0}=0$ satisfies (5) trivially and we examine $n=1$ as the base case, since the recurrence is defined for $n \geq 2$. Namely, for $a_{1}=-\dot{y}$,

$$
\left|a_{1}^{(l)}(t)\right|=\left|y^{(l+1)}(t)\right| \leq M \frac{l !^{\alpha}}{R^{l}} \leq \frac{M}{R^{l}} l !^{\alpha}(l+1)^{\alpha},
$$

and the last inequality is strict when $l>0$. By inductive hypothesis, we assume now that (5) holds for all $i=$ $0,1, \ldots, n-1$ and prove that it must also hold for $i=n$. Taking an absolute value and $l$ time derivatives of (3), after the triangle inequality we have

$$
\begin{aligned}
\left|a_{n}^{(l)}\right| \leq & \left|a_{n-2}^{(l+1)}\right|+\nu\left|a_{n-1}^{(l)}\right|+\sum_{m=0}^{l}\left(\begin{array}{c}
l \\
m
\end{array}\right)\left|a_{n-1}^{(l-m)}\right|\left|y^{(m+1)}\right| \\
& +\rho \sum_{k=0}^{n-2} \sum_{r=0}^{l}\left(\begin{array}{c}
n-2 \\
k
\end{array}\right)\left(\begin{array}{c}
l \\
r
\end{array}\right)\left|a_{n-2-k}^{(r)}\right|\left|a_{k}^{(l-r)}\right| .
\end{aligned}
$$

The first two terms in (6) can be majorized using (5) as

$$
\begin{aligned}
\left|a_{n-2}^{(l+1)}\right| & \leq \frac{M A^{n-3}}{R^{l+n-2}} \frac{(l+n-1) !^{\alpha}}{(n-2) !^{\alpha-1}} \\
& =\frac{M A^{n-1}}{R^{l+n-1}} \frac{(l+n) !^{\alpha}}{n !^{\alpha-1}}\left[\frac{R}{A^{2}} \frac{(n(n-1))^{\alpha-1}}{(l+n)^{\alpha}}\right], \\
\nu\left|a_{n-1}^{(l)}\right| & \leq \nu \frac{M A^{n-2}}{R^{l+n-2}} \frac{(l+n-1) !^{\alpha}}{(n-1) !^{(-1}} \\
& =\frac{M A^{n-1}}{R^{l+n-1}} \frac{(l+n) !^{\alpha}}{n !^{\alpha-1}}\left[\nu \frac{R}{A} \frac{n^{\alpha-1}}{(l+n)^{\alpha}}\right] .
\end{aligned}
$$

The third term in (6) is majorized as

$$
\begin{aligned}
\sum_{m=0}^{l}\left(\begin{array}{c}
l \\
m
\end{array}\right)\left|a_{n-1}^{(l-m)}\right|\left|y^{(m+1)}\right| & \\
\leq & \sum_{m=0}^{l}\left(\begin{array}{c}
l \\
m
\end{array}\right) \frac{M^{2} A^{n-2}}{R^{l+n-m-2} R^{m}} \frac{(l+n-m-1) !^{\alpha} m !^{\alpha}}{(n-1) !^{\alpha-1}} \\
= & \frac{M A^{n-1}}{R^{l+n-1}} \frac{(l+n) !^{\alpha}}{n !^{\alpha-1}}\left[\frac{M R}{A} \frac{n !^{\alpha-1}}{(l+n) !^{\alpha}} \times\right. \\
& \left.\frac{1}{(n-1) !^{\alpha-1}} \sum_{m=0}^{l}\left(\begin{array}{c}
l \\
m
\end{array}\right)(l+n-m-1) !^{\alpha} m !^{\alpha}\right] .
\end{aligned}
$$

Using Lemma 2 and Lemma 3, we can bound the term

$$
\begin{aligned}
\sum_{m=0}^{l}\left(\begin{array}{c}
l \\
m
\end{array}\right)(l+n-m-1) !^{\alpha} m !^{\alpha} \leq \\
\quad\left[\sum_{m=0}^{l}\left(\begin{array}{c}
l \\
m
\end{array}\right)(l+n-m-1) ! m !\right]^{\alpha}=\left[\frac{(n-1) !(n+l) !}{n !}\right]^{\alpha}
\end{aligned}
$$

resulting in

$$
\sum_{m=0}^{l}\left(\begin{array}{c}
l \\
m
\end{array}\right)\left|a_{n-1}^{(l-m)}\right|\left|y^{(m+1)}\right| \leq \frac{M A^{n-1}}{R^{l+n-1}} \frac{(l+n) !^{\alpha}}{n !^{\alpha-1}}\left[\frac{M R}{A n}\right] .
$$

The fourth (nonlinear) term in (6) is majorized as

$$
\begin{aligned}
& \rho \sum_{k=0}^{n-2} \sum_{r=0}^{l}\left(\begin{array}{c}
n-2 \\
k
\end{array}\right)\left(\begin{array}{l}
l \\
r
\end{array}\right)\left|a_{n-2-k}^{(r)}\right|\left|a_{k}^{(l-r)}\right| \\
& \leq \rho \sum_{k=0}^{n-2} \sum_{r=0}^{l}\left(\begin{array}{c}
n-2 \\
k
\end{array}\right)\left(\begin{array}{l}
l \\
r
\end{array}\right) \times \\
& \frac{M^{2} A^{n-4}}{R^{l+n-4}} \frac{(n+r-k-2) !^{\alpha}(l+k-r) !^{\alpha}}{(n-k-2) !^{\alpha-1} k !^{\alpha-1}} \\
& \leq \frac{M A^{n-1}}{R^{l+n-1}} \frac{(l+n) !^{\alpha}}{n !^{\alpha-1}} \times \\
& {\left[\frac{\rho M R^{3}}{A^{3}} \frac{n !^{\alpha-1}}{(l+n) !^{\alpha}} \sum_{k=0}^{n-2}\left(\begin{array}{c}
n-2 \\
k
\end{array}\right) \frac{1}{(n-k-2) !^{\alpha-1} k !^{\alpha-1}}\right.} \\
& \left.\times\left\{\sum_{r=0}^{l}\left(\begin{array}{l}
l \\
r
\end{array}\right)(n+r-k-2) !(l+k-r) !\right\}^{\alpha}\right] \text {, }
\end{aligned}
$$

where the last inequality makes use of Lemma 3. Using 
Lemma 2 we have

$$
\begin{aligned}
& \sum_{k=0}^{n-2}\left(\begin{array}{c}
n-2 \\
k
\end{array}\right) \frac{1}{(n-k-2) !^{\alpha-1} k !^{\alpha-1}} \times \\
& \left\{\sum_{r=0}^{l}\left(\begin{array}{l}
l \\
r
\end{array}\right)(n+r-k-2) !(l+k-r) !\right\}^{\alpha} \\
& =\sum_{k=0}^{n-2}\left(\begin{array}{c}
n-2 \\
k
\end{array}\right) \frac{1}{(n-k-2) !^{\alpha-1} k !^{\alpha-1}} \times \\
& \left\{\frac{k !(n-k-2) !(n+l-1) !}{(n-1) !}\right\}^{\alpha} \\
& =\sum_{k=0}^{n-2}\left(\begin{array}{c}
n-2 \\
k
\end{array}\right)(n-k-2) ! k !\left\{\frac{(n+l-1) !}{(n-1) !}\right\}^{\alpha} \\
& =\sum_{k=0}^{n-2}(n-2) !\left\{\frac{(n+l-1) !}{(n-1) !}\right\}^{\alpha} \\
& =(n-1)(n-2) !\left\{\frac{(n+l-1) !}{(n-1) !}\right\}^{\alpha}=\frac{(n+l-1) !^{\alpha}}{(n-1) !^{\alpha-1}},
\end{aligned}
$$

resulting in

$$
\begin{aligned}
\rho \sum_{k=0}^{n-2} & \sum_{r=0}^{l}\left(\begin{array}{c}
n-2 \\
k
\end{array}\right)\left(\begin{array}{c}
l \\
r
\end{array}\right)\left|a_{n-2-k}^{(r)}\right|\left|a_{k}^{(l-r)}\right| \\
& \leq \frac{M A^{n-1}}{R^{l+n-1}} \frac{(l+n) !^{\alpha}}{n !^{\alpha-1}}\left[\frac{\rho M R^{3}}{A^{3}} \frac{n !^{\alpha-1}}{(l+n) !^{\alpha}} \frac{(n+l-1) !^{\alpha}}{(n-1) !^{\alpha-1}}\right] \\
& =\frac{M A^{n-1}}{R^{l+n-1}} \frac{(l+n) !^{\alpha}}{n !^{\alpha-1}}\left[\frac{\rho M R^{3}}{A^{3} n}\left(\frac{n}{n+l}\right)^{\alpha}\right] .
\end{aligned}
$$

Collecting the terms for (6) and noticing that for $n \geq 1$, $l \geq 0$, and $\alpha \geq 0,\left[\frac{n}{n+l}\right]^{\alpha} \leq 1$ holds, we have

$$
\begin{aligned}
\left|a_{n}^{(l)}\right| \leq \frac{M A^{n-1}}{R^{l+n-1}} \frac{(l+n) !^{\alpha}}{n !^{\alpha-1}} \times & \\
& {\left[\frac{R}{A^{2}} \frac{(n-1)^{\alpha-1}}{n}+\frac{(\nu+M) R}{A n}+\frac{\rho M R^{3}}{A^{3} n}\right] . }
\end{aligned}
$$

The terms in the square brackets are bounded using

$$
\max _{n \geq 2, \alpha \in[1,2]} \frac{(n-1)^{\alpha-1}}{n}=\left.\frac{(n-1)^{1}}{n}\right|_{(n \geq 2)} \leq 1 .
$$

With these bounds, we have

$$
\begin{aligned}
\left|a_{n}^{(l)}\right| \leq & \frac{M A^{n-1}}{R^{l+n-1}} \frac{(l+n) !^{\alpha}}{n !^{\alpha-1}} \\
& {\left[\frac{1}{R}\left(\frac{R}{A}\right)^{2}+\frac{(\nu+M)}{2}\left(\frac{R}{A}\right)+\frac{\rho M}{2}\left(\frac{R}{A}\right)^{3}\right] . }
\end{aligned}
$$

Given $(M, R, \rho, \nu)$, the parameter $A$ is chosen such that

$$
\left[\frac{1}{R}\left(\frac{R}{A}\right)^{2}+\frac{(\nu+M)}{2}\left(\frac{R}{A}\right)+\frac{\rho M}{2}\left(\frac{R}{A}\right)^{3}\right]=1,
$$

implying that (5) is proven by induction. Using (5) and the Cauchy-Hadamard Formula, the radius of convergence is given by

$$
\begin{aligned}
\frac{1}{\limsup _{n \rightarrow \infty}\left|a_{n} / n !\right|^{1 / n}} & \geq\left[\lim _{n \rightarrow \infty}\left|\frac{M A^{n-1}}{R^{n-1}}\right|^{1 / n}\right]^{-1} \\
& =\lim _{n \rightarrow \infty} \frac{R}{A}\left[\frac{A}{M R}\right]^{1 / n}=\frac{R}{A} .
\end{aligned}
$$

Denoting this lower bound on the radius of convergence as $\eta \equiv R / A$ and substituting into (7) yields (4). Existence and uniqueness of the positive root $\eta=\eta^{*}$ are now proven. Given $(M, R, \nu, \rho)>0$, define the positive, analytic and strictly increasing function $\eta \mapsto f(\eta)$ as

$$
f(\eta)=\left(\frac{\rho M}{2}\right) \eta^{3}+\left(\frac{1}{R}\right) \eta^{2}+\left(\frac{\nu+M}{2}\right) \eta .
$$

The positive root $\eta^{*}$ of the equation $f\left(\eta^{*}\right)-1=0$ exists and is unique since $(f(\cdot)-1)(\eta)$ is analytic and strictly increases from -1 to $+\infty$ as $\eta$ grows from 0 to $+\infty$.

Remark 1: We give here analytic expressions of the first five coefficients of the series (2) so one can see how the successive derivatives of $y$ appear through recurrence (3)

$$
\begin{aligned}
a_{1}= & -\dot{y} \\
a_{2}= & -\dot{y}(\nu+\dot{y}) \\
a_{3}= & -\ddot{y}+\dot{y}^{3}-\nu^{2} \dot{y} \\
a_{4}= & -\ddot{y}(2 \nu+\dot{y})-\dot{y}^{4}+\nu \dot{y}^{3}+\left(\nu^{2}+2 \rho\right) \dot{y}^{2}-\nu^{3} \dot{y} \\
a_{5}= & -y^{(3)}-3 \nu^{2} \ddot{y}+\dot{y}^{5}-2 \nu \dot{y}^{4}+4 \rho \dot{y}^{3} \\
& +\left(4 \ddot{y}+2 \nu\left(\nu^{2}+4 \rho\right)\right) \dot{y}^{2}+\left(\nu \ddot{y}^{2}-\nu^{4}\right) \dot{y} .
\end{aligned}
$$

\section{Parameterizations of Radius of Convergence}

This section is concerned with a parametric lower bound on $\eta^{*}$. The bound is achieved using the following lemma.

Lemma 1: For all $a, b, c$ strictly positive real parameters, the unique positive root $\eta^{0}$ of

$$
a \eta^{3}+b \eta^{2}+c \eta-1=0
$$

is lower bounded by

$$
\frac{-c+\sqrt{c^{2}+4(a / c+b)}}{2(a / c+b)} .
$$

Proof: The function $\eta \mapsto a \eta^{3}+b \eta^{2}+c \eta-1$ is analytic and strictly increases from -1 to $+\infty$ as $\eta$ grows from 0 to $+\infty$. Define $h_{1}(\eta)=a \eta^{3}+b \eta^{2}, h_{2}(\eta)=1-c \eta$. The graphs of $h_{1}$ and $h_{2}$ intersect at $\eta^{0}$. Since $h_{1}>0$ on $] 0,+\infty[$ it is clear that $\eta^{0}<1 / c$.

On $] 0,1 / c\left[\right.$ it is easy to check that $h_{1}(\eta)<(a / c+b) \eta^{2}$. On this interval $h_{1}$ is a strictly increasing function while $h_{2}$ is strictly decreasing. Let $\hat{\eta}$ be the unique positive root of $(a / c+b) \eta^{2}=1-c \eta$ and we get that $h_{1}(\hat{\eta})<h_{2}(\hat{\eta})$, yielding $\hat{\eta}<\eta^{0}$. Finally

$$
\eta^{0}>\frac{-c+\sqrt{c^{2}+4(a / c+b)}}{2(a / c+b)} .
$$


When $a=\rho M / 2, b=1 / R$ and $c=(\nu+M) / 2$, it is clear that $\eta^{0}$ corresponds to $\eta^{*}$. We now give the main analytic result of the paper regarding convergence of the proposed solution, making use of the last lower bound.

Theorem 2: Given $\nu, \rho>0$ and assuming that $\dot{y} \in$ $C^{\infty}[0, T]$ is Gevrey of order $(\alpha-1)$ for $1 \leq \alpha \leq 2$, i.e.

$$
\exists M, R>0 \text { such that }\left|y^{(l+1)}(t)\right| \leq M \frac{l !^{\alpha}}{R^{l}}
$$

$\forall l=0,1,2, \ldots$, the radius of convergence of the series (2) is greater than $\hat{\eta}$ as given by formula (10)

This result can be complemented with tighter bounds for the special case when $\rho$ is large and $\nu$ is small (see [2]).

\section{Numerical Simulations}

Assume one wants to grow the liquid column from an initial length 1.0 with uniform zero temperature to a final length 2.0 with uniform zero temperature. This problem is challenging since the actuation is at the fixed boundary, a surface opposite to the melting interface. As such, the openloop control must compensate for both the energy loss due to melting at the interface and due to the diffusion and reaction terms.

For practical purposes of course the series solution (2) is truncated for implementation. For steady-state to steady-state column growth, the interface motion $y(t)$ is defined as

$$
y(\tau)= \begin{cases}L+\Delta L & \text { if } \tau \geq T, \\ L+\Delta L g(\tau / T) & \text { if } T>\tau>0, \\ L & \text { if } \tau \leq 0\end{cases}
$$

where

$$
g(\tau)=\frac{f(\tau)}{f(\tau)+f(1-\tau)}, \tau \in[0,1]
$$

and

$$
f(\tau)= \begin{cases}e^{-\frac{1}{\tau}} & \text { if } \tau>0 \\ 0 & \text { if } \tau \leq 0\end{cases}
$$

The function $y$ defines a smooth transition from $L$ to $L+\Delta L$ in liquid column length ${ }^{1}$, with Gevrey constants for $\dot{y}$ equal to

$$
M=\frac{\Delta L e^{2}}{T \sqrt{2 \pi}}, R=\frac{T}{4} .
$$

We take the first 10 terms to approximate the solution $u(x, t)$. Steady-state to steady-state simulations are considered and it is easy to verify $\psi \equiv 0$. Figure 4 shows the temperature profile for $T=100, \rho=1.2, \nu=0.5, L=1.0$, and $L+$ $\Delta L=2.0$. The analytic bound in (10) yields $\hat{\eta}=2.0619$ which guarantees convergence over the desired domain. For this case, we numerically computed $\eta^{*}=2.2506$, showing the relative conservatism of (10) quantitatively. It is to be

\footnotetext{
${ }^{1}$ The function chosen above is based upon an unpublished work of François Malrait done at École des Mines (see [2])
}

noted that as $T$ gets large $\eta^{*}$ tends to $2 / \nu$ and so does its lower bound $\hat{\eta}$.

$\mathrm{u}(\mathrm{x}, \mathrm{t})$ temperature

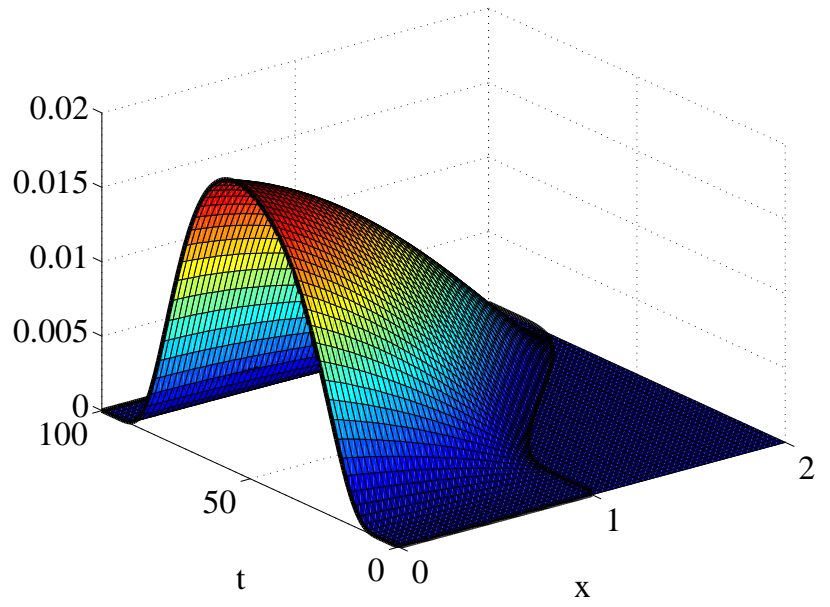

Fig. 4. Temperature profile for transition from column length 1 to 2.

The derivation of the Gevrey constants for $\dot{y}$ above and more simulation results are detailed in [2].

\section{CONClusions And Future Work}

In this paper, we have considered boundary control of a simplified model of crystal growth. The problem includes two technical difficulties: the moving boundary and a quadratic reaction term. When combined, these issues make convergence substantially more difficult to study. We derived conservative results that can indeed be used in practice, as shown in the simulation section. The solution we propose must be used with caution or a physical requirement of the model, namely non-negativity of the temperature in the liquid phase, may not be satisfied. It has been shown in [2] that this physical requirement is asymptotically satisfied as the transient time from one stead-state to another becomes large.

An issue for future work is the study of approximate stabilizability of the model studied in this paper. Replacing the zero initial condition $u(x, 0)=0$ by an arbitrary initial condition $u(x, 0)=\psi(x)$, is it still possible to steer the system (at least approximately) to zero in finite time, i.e. $u(x, T) \approx 0$ ?

Usually, such a result arises from a projection of the initial condition onto the formal series expression (refer to [10]). Straightforward conditions are thus available for formal series of the form $u(x, t)=\sum_{i=0}^{\infty} y^{(n)}(t) \frac{x^{2 n}}{(2 n) !}$ using the fact that the set of polynomials $x^{2 n}, n \in \mathbb{N}$ is dense in the set of $L^{2}$ functions. In a simple manner, the conditions result in specified values for all the derivatives of $y$ at the initial time 0 .

In our case the series expansion is much different. Due to the moving boundary and the nonlinear effect, no simple 


$$
\hat{\eta}=\frac{1}{4(\rho R M+\nu+M)}\left(-R(\nu+M)^{2}+\sqrt{R^{2}(\nu+M)^{4}+16\left(\rho M R^{2}(\nu+M)+R(\nu+M)^{2}\right)}\right)
$$

Fig. 3. Bound for Theorem 2.

identification between the $\left(a_{i}\right)$ coefficients and the derivatives of $y$ can be achieved. Moreover, both even and odd polynomials appear for most derivatives of $y$, ruling out classical density results, e.g. Müntz-Szasz theorem [16]. All this makes the situation more convoluted and difficult to handle. This point is currently under investigation.

\section{APPENDIX: TECHNICAL LEMMAS}

\section{Lemma 2:}

$$
\frac{i ! j !(i+j+l+1) !}{(i+j+1) !}=\sum_{r=0}^{l}\left(\begin{array}{l}
l \\
r
\end{array}\right)(j+r) !(i+l-r) !
$$

for $i, j, l \geq 0$.

Proof: This result directly follows from the ChuVandermonde identity [14]. Details can be found in [2].

Lemma 3: For $\alpha, c_{k} \geq 1$ and $b_{k} \geq 0, k=0,1, \ldots, l$,

$$
\sum_{k=0}^{l} c_{k}\left(b_{k}\right)^{\alpha} \leq\left(\sum_{k=0}^{l} c_{k} b_{k}\right)^{\alpha}, \quad l \geq 0 .
$$

Proof: ${ }^{k=0}$ The proof is an easy extension of that for the case $c_{k}=1, \forall k=0,1, \ldots, l$, given in [7].

\section{REFERENCES}

[1] J. R. Cannon. The one-dimensional heat equation, volume 23 of Encyclopedia of Mathematics and its applications. Addison-Wesley Publishing Company, 1984.

[2] W. B. Dunbar, N. Petit, P. Rouchon, and Ph. Martin. Motion planning for a nonlinear Stefan problem. ESAIM: Control, Optimisation and Calculus of Variations, 9:275-296, February 2003.

[3] M. Fila and P. Souplet. Existence of global solutions with slow decay and unbounded free boundary for a superlinear Stefan problem. Interfaces and Free Boundaries, 3:337-344, 2001.

[4] M. Fliess, J. Lévine, Ph. Martin, and P. Rouchon. Flatness and defect of nonlinear systems: introductory theory and examples. Int. J. Control, 61(6):1327-1361, 1995.

[5] M. Fliess, J. Lévine, Ph. Martin, and P. Rouchon. A Lie-Bäcklund approach to equivalence and flatness of nonlinear systems. IEEE Trans. Automat. Control, 44:922-937, 1999.

[6] A. Friedman and B. Hu. A Stefan problem for multidimensional reaction-diffusion systems. SIAM J. Math. Anal., 27:1212-1234, 1996.

[7] M. Gevrey. La nature analytique des solutions des équations aux dérivées partielles. Ann. Sci. École Norm. Sup., 25:129-190, 1918.
[8] C. D. Hill. Parabolic equations in one space variable and the non-characteristic Cauchy problem. Comm. Pure Appl. Math., 20:619-633, 1967.

[9] Chen Hua and L. Rodino. General theory of partial differential equations and microlocal analysis. In Qi MinYou and L. Rodino, editors, Proc. of the workshop on General theory of PDEs and Microlocal Analysis, International Centre for Theoretical Physics, Trieste, pages 6-81. Longman, 1995.

[10] B. Laroche, Ph. Martin, and P. Rouchon. Motion planing for the heat equation. Int. Journal of Robust and Nonlinear Control, 10:629-643, 2000.

[11] A. F. Lynch and J. Rudolph. Flatness-based boundary control of a nonlinear parabolic equation modelling a tubular reactor. In A. Isidori, F. Lamnabhi-Lagarrigue, and W. Respondek, editors, Lecture Notes in Control and Information Sciences 259: Nonlinear Control in the Year 2000, volume 2, pages 45-54. Springer, 2000.

[12] M. B. Milam, K. Mushambi, and R. M. Murray. A new computational approach to real-time trajectory generation for constrained mechanical systems. In IEEE Conference on Decision and Control, 2000.

[13] N. Petit, M. B. Milam, and R. M. Murray. A new computational method for optimal control of a class of constrained systems governed by partial differential equations. In Proc. of the 15th IFAC World Congress, 2002.

[14] M. Petkovsek, H.S. Wilf, and D. Zeilberger. $A=B$. Wellesley, 1996.

[15] L.I. Rubinstein. The Stefan problem, volume 27 of Translations of mathematical monographs. AMS, Providence, Rhode Island, 1971.

[16] W. Rudin. Real and Complex Analysis. McGraw-Hill International Editions, third edition, 1987. 